\title{
Perspective
}

\section{Double target concept for smoking cessation}

\author{
Jie $\mathrm{WU}^{1,2, *}$ \\ ${ }^{1}$ Shantou University Medical College, Shantou 515041, China; ${ }^{2}$ Barrow Neurological Institute, St Joseph's Hospital and Medical \\ Center, Phoenix AZ 85013, USA
}

\begin{abstract}
Tobacco use is estimated to be the largest single cause of premature death in the world. Nicotine is the major addictive substance in tobacco products. After cigarette smoking, nicotine quickly acts on its target, nicotinic acetylcholine receptors (nAChRs), which are widely distributed throughout the mammalian central nervous system and are expressed as diverse subtypes on cell bodies, dendrites and/or nerve terminals. Through the nAChRs in brain reward circuits, nicotine alters dopaminergic (DA) neuronal function in the ventral tegmental area (VTA) and increases dopamine release from VTA to nuclear accumbens (NA), which leads to nicotine reward, tolerance and dependence. After quitting smoking, smokers experience withdrawal symptoms, including depression, irritability, difficulty concentrating or sleeping, headache, and tiredness. Recently, evidence has been accumulated to reveal the molecular and cellular mechanisms of nicotine reward, tolerance and dependence. The outcomes of these investigations provide pharmacological basis for smoking cessation. Here, I briefly summarize recent advancements of our understanding of nicotine reward, tolerance and dependence. Based on these understandings, I propose a double target hypothesis, in which nAChRs and dopamine release process are two important targets for smoking cessation. Dysfunction of nAChRs (antagonism or desensitization) is crucial to abolish nicotine dependence and the maintenance of an appropriate level of extracellular dopamine eliminates nicotine withdrawal syndromes. Therefore, the medications simultaneously act on these two targets should have the desired effect for smoking cessation. I discuss how to use this double target concept to interpret recent therapies and to develop new candidate compounds for smoking cessation.
\end{abstract}

Keywords: nicotine; nicotine addiction; nicotine reward; nicotine dependence; nicotine withdrawal syndromes; nicotinic acetylcholine receptor; smoking cessation

Acta Pharmacologica Sinica (2010) 31: 1015-1018; doi: 10.1038/aps.2010.137; published online 16 Aug 2010

Nicotine is a potent addictive substance in the tobacco that is thought to promote the use of tobacco products by about onequarter of the world's population. Tobacco use is the leading preventable cause of disease, disability, and death. Cigarette smoking results in more than 400000 premature deaths each year - about 1 in every 5 US deaths. Economically, more than $\$ 75$ billion per year of total US healthcare costs is attributable directly to smoking. China is the world's largest producer and consumer of tobacco. It estimates that there are 0.35 billion cigarette smokers in China. Economically, more than \$166 billion per year of total Chinese healthcare costs are attributable directly to smoking-associated diseases. Therefore, there is a considerable need to reduce the population of smokers. Unfortunately, nicotine addiction severely confounds attempts to end tobacco product use.

Nicotine addiction has been clinically delineated into two specific diagnosable disorders: dependence and withdrawal

\footnotetext{
* To whom correspondence should be addressed.

E-mail jie.wu@chw.edu

Received 2010-05-30 Accepted 2010-07-20
}

symptoms. Nicotine dependence refers to the maladaptive and chronic use of tobacco that meets the same types of criteria that are applied to other forms of drug addiction. Recent research has revealed two important features of nicotine addiction: (1) nAChRs play a critical role in developing nicotine addiction, and (2) nicotine addiction is a dynamic process including different stages such as nicotine-induced reward, tolerance, dependence and withdrawal-relapse symptoms ${ }^{[1]}$. Nicotine reward means that nicotine, acting on brain nAChRs, stimulates brain reward-associated circuits, which allows the smoker to be in a euphoric state. Dopamine is one of the key neurotransmitters actively involved within the reward circuits in the brain. Accumulating lines of evidence indicate that nicotine increases DA release from VTA to NA, which represents its nature of reward and intense addictive qualities.

Mounting evidence demonstrates that nAChR subtypes with different distributions within reward circuits mediate nicotine reward. Dopaminergic (DA) neurons in the VTA express diverse nAChR subunits, including a3-a7 and $\beta 2-\beta 4^{[2-7]}$, which can combine to form at least two pharmacologically distinct $\mathrm{nAChR}$ subtypes. One of these functionally 
distinct $\mathrm{nAChRs}$ is homomeric a7-nAChRs, which are mainly expressed on glutamatergic presynaptic terminals, where they mediate nicotine-induced increase of glutamate release onto DA neurons ${ }^{[6]}$. Another group of nAChRs is non-a7-nAChRs, which seems to be more complex. For example, electrophysiological studies, combined with single-cell RT-PCR technique, show a $4 a 6 a 5(\beta 2)_{2}$ or $a 4 a 5(\beta 2)_{2}$ combinations of nAChRs in midbrain reward center ${ }^{[6]}$. Immunoprecipitation approaches using $\mathrm{nAChR}$ subunit knockout mice show that functional a $6 \beta 2-n A C h R s$ are mainly located on DA neuronal terminals, whereas a $4 \beta 2$-nAChRs represent the majority of functional heteromeric nAChRs on DA neuron somata ${ }^{[8]}$. Interestingly, GABAergic neurons located in the VTA likely express relatively-simple nAChR subtypes, mainly $(\alpha 4)_{2}(\beta 2)_{3}-\mathrm{nAChRs}{ }^{[6]}$ and these $\alpha 4 \beta 2 \mathrm{nAChRs}$ contribute to cholinergic modulation of GABA tonic release onto DA neurons ${ }^{[9]}$. Recently, we have characterized three functional subtypes (ID, IID, IIID) of nAChRs in VTA DA neurons ${ }^{[7]}$ that may mediate nicotine reward and dependence. For example, nicotine, in the same concentrations and time ranges as obtained from cigarette smoking, enhances glutamatergic excitation in the VTA DA neurons by increasing glutamate release via stimulating presynaptic a7-nAChRs ${ }^{[10]}$. Nicotine also activates then desensitizes $\alpha 4 \beta 2-n A C h R s$ on both DA and GABAergic neurons ${ }^{[9]}$. Since cholinergic innervations in GABAergic neurons have much higher density than that in DA neurons ${ }^{[11]}$, the nicotineinduced a $4 \beta 2$-nAChR desensitization (after brief activation) mainly decreases GABA release onto DA neurons, and consequently increases DA neuronal activity. It has been proposed that nicotine-induced desensitization is at least one of the major mechanisms for nicotine addiction ${ }^{[12-14]}$. In the VTA, nicotine activates ( $\alpha 7-$ ) and/or desensitizes ( $\alpha 4 \beta 2)$ nAChRs, and in turn increases DA neuronal activity. The short-lived direct excitation of the DA neurons coupled with the enhanced glutamatergic afferent activity provides the presynaptic and postsynaptic coincidence necessary to initiate synaptic potentiation and plasticity $(\mathrm{LTP})^{[15,16]}$. Taken together, these synaptic events lead to a relatively long-lasting heightened activity of midbrain DA neurons, which release more DA to NA and results in positive reinforcement. The nicotine reward is an important early event to initiate and develop to nicotine dependence.

As mention before, nicotine, in addition to activating nAChRs, also desensitizes them ${ }^{[17]}$, which plays an important role in the developing nicotine tolerance and dependence. After repetitive exposure to nicotine, $\mathrm{nAChR}$ desensitization represents a low functional status of nAChRs in the presence of low level of nicotine, and the recovery from receptor desensitization is relative slow. Under this desensitization condition, the same concentrations of nicotine are not enough to induce the same level of reward signals (the level of extracellular DA in the NA), which consequently causes nicotine tolerance. On one hand, to overcome nicotine tolerance (to get the same level of reward stimulation), smokers increase cigarette smoked per day, which results in the nicotine dependence. On the other hand, nAChR desensitization triggers receptor upregulation, and the upregulated nAChRs imbalances cholinergic signaling and modulation, which causes withdrawal symptoms when smoker quits cigarette smoking.

In summary, nicotine addiction is the major reason why smokers continue to rely on tobacco products. Nicotine dependence and withdrawal symptoms are two pathophysiological changes brought on by cigarette smoking. Based on these lines of evidence, I propose a "double target" hypothesis to interpret pharmacotherapuetic mechanisms of smoking cessation. In this hypothesis, I assume that the medications that eliminate both nicotine dependence and withdrawal-relapse will exert more desired effect for smoking cessation (Figure 1). To reduce nicotine dependence, medications should block nAChRs (in particular a $4 \beta 2-n A C h R s$ ), while to eliminate withdrawal-relapse, they also should appropriately (mildly) increase extracellular DA level in the NA.

Using this double target concept, one can explain pharmacological mechanisms of existing smoking cessation medications. For instance, recent market drugs available for smoking cessation are bupropion and varenicline ${ }^{[18]}$. Bupropion is used to treat mental depression, but it is also used as part of a support program to help people stop smoking ${ }^{[19]}$. This medicine exhibits double target properties since it not only blocks dopamine transporter (maintain a mildly elevated level of extracellular DA in the NA), but also blocks nAChRs, in particular a $4 \beta 2-$ $n A C h R s^{[19-21]}$. Varenicline is another example of a smoking

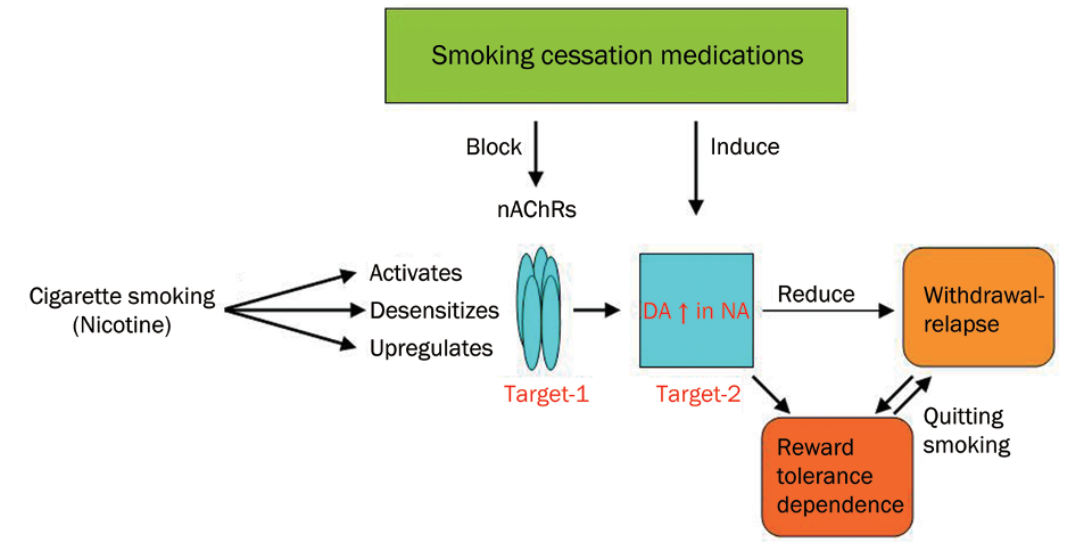

Figure 1. Scheme of double target hypothesis for smoking cessation. Target 1 is the nAChRs that is critical for nicotine reward, tolerance and dependence, while target 2 is the maintenance of mild extracellular DA level in the rewarding circuit (NA), which eliminates withdrawal-relapse syndromes. 
cessation medication ${ }^{[22]}$ that matches the double target concept. It is a partial agonist at $\alpha 4 \beta 2-n A C h R s$, and is also a full agonist at $\alpha 7 \mathrm{nAChRs}{ }^{[23]}$. As a partial agonist of $\alpha 4 \beta 2-\mathrm{nAChRs}$, varenicline mimics nicotinic effect to activate/desensitize its first target, a4 $32-n A C h R$, and diminishes nicotine dependence. In addition, as a full agonist at $\alpha 7 \mathrm{nAChRs,} \mathrm{it} \mathrm{also} \mathrm{mimics} \mathrm{nico-}$ tinic stimulation on its second target, presynaptic a7-nAChR, to increase glutamate release onto DA neurons in the VTA, which in turn appropriately increases DA release from the VTA to NA, and eliminates withdrawal symptoms.

Based on this double target concept, one also can develop new medications for smoking cessation. Recently, my laboratory has developed two different compounds that exhibit high potential to be novel smoking cessation medications. One group of compounds is called tetrahydroprotoberberine analogs (THPBs) which include tetrahydroberberine (THB), l-stepholidine (1-SPD) and 1-hydroparmatine (1-THP). THPBs are purified from several Chinese herbs in the magnoliidae superorder. Mounting evidence indicates that THPBs exhibit dopamine receptor $\left(D_{2}\right)$ antagonist effects on sedation, hypnosis, antinociception, anti-schizophrenia and anti-hypertension ${ }^{\text {[24-27]. }}$ The major pharmacological targets for THPBs are dopamine receptors, and THPBs exhibit D1 partial agonist and D2 antagonist effects. As a D2 receptor antagonist in VTA DA neurons, systemic exposure to THPBs increase DA neuronal firing by blocking D2 auto-receptors on DA neurons and increase DA level in NA. It has been reported that 1-THP effectively eliminates heroin addiction in human ${ }^{[28]}$, suggesting that THPBs may have a potential for treating drug addiction. Recent research in my laboratory also shows that THPBs potently inhibit either human a $4 \beta 2-n A C h R s$ heterologously expressed in SH-EP1 cell line or rodent a $4 \beta 2-n A C h R s$ in midbrain DA neurons. This double-target feature suggests that THPBs are good candidates that can be developed into new smoking cessation medications.

Another compound we are working on is iptakalim hydrochloride (Ipt). Ipt was initially designed and synthesized as an antihypertensive drug ${ }^{[29]}$. It is a small, water soluble molecule that freely penetrates the blood-brain barrier and has minimal toxic side effects following long-term systemic administration $^{[30]}$. Possible pharmacological mechanisms underlying its antihypertensive action include $\mathrm{K}_{\mathrm{ATP}}$ channel activation and endothelin antagonism. Tests in a variety of in vivo and in vitro ischemia and Parkinson's disease models indicate that Ipt has neuroprotective effects ${ }^{[30-32]}$. Furthermore, Ipt can potentially prevent drug addiction since it inhibits cocaine challengeinduced enhancement of dopamine release in rat $\mathrm{NA}^{[33]}$. The major pharmacological target of Ipi is thought to the cytoplasmic and/or mitochondrial $K_{\mathrm{ATP}}$ channels ${ }^{[30]}$. My laboratory has evaluated the effects of Ipt on $\mathrm{nAChR}$ function and found that Ipt potently blocks a $4 \beta 2-n A C h R s$ heterologously expressed in human SH-EP1 cell line ${ }^{[34]}$ or natively expressed in rat midbrain DA neurons ${ }^{[35]}$. Currently, we have also found that Ipt significantly prevents systemic nicotine-induced behavioral (locomotor activity) sensitization, further confirmed its $\mathrm{AAChR}$ antagonism (Wu et al, unpublished data). Interestingly, sys- temic injections of Ipt along (30 $\mathrm{mg} \cdot \mathrm{kg}^{-1} \cdot \mathrm{day}^{-1}$, ip for 7 days) increase rat locomotor activity (Wu et al, unpublished data), suggesting that on one hand, Ipt can block a $4 \beta 2-n A C h R$ function, and on the other hand, it also can appropriately increase DA level at NA although the reason and mechanisms of this effect are still unclear. Nevertheless, Ipt also exhibits doubletarget feature and it likely can be developed as a novel smoking cessation medication.

In conclusion, nicotine addiction is a complex brain disorder, which involves functional alterations in multiple brain circuits and exhibits different stages. Based on the new concept of network pharmacology ${ }^{[36,37]}$, I propose a double target hypothesis, which may help to understand the pharmacotherapeutic mechanisms of smoking cessation, and also help to develop new medications for quitting tobacco use.

\section{Acknowledgements}

Work toward this project was supported by grants from the Arizona Biomedical Research Commission, the Institute for Mental Health Research, and Philip Morris International through their External Research Program.

\section{References}

1 Brennan K, Lea R, Fitzmaurice P, Truman P. Review: Nicotinic receptors and stages of nicotine dependence. J Psychopharmacol 2010; 24: 793-808.

2 Sargent PB. The diversity of neuronal nicotinic acetylcholine receptors. Annu Rev Neurosci 1993; 16: 403-43.

3 Charpantier E, Barneoud P, Moser P, Besnard F, Sgard F. Nicotinic acetylcholine subunit mRNA expression in dopaminergic neurons of the rat substantia nigra and ventral tegmental area. Neuroreport 1998; 9: 3097-101.

4 Sgard F, Charpantier E, Barneoud P, Besnard F. Nicotinic receptor subunit mRNA expression in dopaminergic neurons of the rat brain. Ann N Y Acad Sci 1999; 868: 633-5.

5 Le Novere N, Corringer PJ, Changeux JP. The diversity of subunit composition in nAChRs: evolutionary origins, physiologic and pharmacologic consequences. J Neurobiol 2002; 53: 447-56.

6 Klink R, de Kerchove d'Exaerde A, Zoli M, Changeux JP. Molecular and physiological diversity of nicotinic acetylcholine receptors in the midbrain dopaminergic nuclei. J Neurosci 2001; 21: 1452-63.

7 Yang K, Hu J, Lucero L, Liu Q, Zheng C, Zhen X, et al. Distinctive nicotinic acetylcholine receptor functional phenotypes of rat ventral tegmental area dopaminergic neurons. J Physiol 2009; 587: 345-61.

8 Champtiaux N, Gotti C, Cordero-Erausquin M, David DJ, Przybylski C, Lena $C$, et al. Subunit composition of functional nicotinic receptors in dopaminergic neurons investigated with knock-out mice. J Neurosci 2003; 23: 7820-9.

9 Mansvelder HD, Keath JR, McGehee DS. Synaptic mechanisms underlie nicotine-induced excitability of brain reward areas. Neuron 2002; 33: 905-19.

10 Wonnacott S, Kaiser S, Mogg A, Soliakov L, Jones IW. Presynaptic nicotinic receptors modulating dopamine release in the rat striatum. Eur J Pharmacol 2000; 393: 51-8.

11 Garzon M, Vaughan RA, Uhl GR, Kuhar MJ, Pickel VM. Cholinergic axon terminals in the ventral tegmental area target a subpopulation of neurons expressing low levels of the dopamine transporter. J Comp Neurol 1999; 410: 197-210.

12 Hulihan-Giblin BA, Lumpkin MD, Kellar KJ. Acute effects of nicotine 
on prolactin release in the rat: agonist and antagonist effects of a single injection of nicotine. J Pharmacol Exp Ther 1990; 252: 15-20.

13 Sharp BM, Beyer HS. Rapid desensitization of the acute stimulatory effects of nicotine on rat plasma adrenocorticotropin and prolactin. J Pharmacol Exp Ther 1986; 238: 486-91.

14 Xiao Y, Fan H, Musachio JL, Wei ZL, Chellappan SK, Kozikowski AP, et al. Sazetidine-A, a novel ligand that desensitizes alpha4beta2 nicotinic acetylcholine receptors without activating them. Mol Pharmacol 2006; 70: 1454-60.

15 Dani JA, Heinemann S. Molecular and cellular aspects of nicotine abuse. Neuron 1996; 16: 905-8.

16 Dani JA, Ji D, Zhou FM. Synaptic plasticity and nicotine addiction. Neuron 2001; 31: 349-52.

17 Wooltorton JR, Pidoplichko VI, Broide RS, Dani JA. Differential desensitization and distribution of nicotinic acetylcholine receptor subtypes in midbrain dopamine areas. J Neurosci 2003; 23: 317685.

18 Fant RV, Buchhalter AR, Buchman AC, Henningfield JE. Pharmacotherapy for tobacco dependence. Handb Exp Pharmacol 2009; (192): 487-510.

19 Paterson NE. Behavioural and pharmacological mechanisms of bupropion's anti-smoking effects: recent preclinical and clinical insights. Eur J Pharmacol 2009; 603: 1-11.

20 Sidhpura N, Redfern P, Wonnacott S. Comparison of the effects of bupropion on nicotinic receptor-evoked $[(3) \mathrm{H}]$ dopamine release from rat striatal synaptosomes and slices. Eur J Pharmacol 2007; 567 : 102-9.

21 Slemmer JE, Martin BR, Damaj MI. Bupropion is a nicotinic antagonist. J Pharmacol Exp Ther 2000; 295: 321-7.

22 Rollema H, Hajos M, Seymour PA, Kozak R, Majchrzak MJ, Guanowsky $\mathrm{V}$, et al. Preclinical pharmacology of the alpha4beta2 nAChR partial agonist varenicline related to effects on reward, mood and cognition. Biochem Pharmacol 2009; 78: 813-24.

23 Mihalak KB, Carroll FI, Luetje CW. Varenicline is a partial agonist at alpha4beta2 and a full agonist at alpha7 neuronal nicotinic receptors. Mol Pharmacol 2006; 70: 801-5.

24 Bian CF, Duan SM, Xing SH, Yu YM, Qin W, Jin GZ, et al. Interaction of analgesics and I-stepholidine. Acta Pharmacol Sin 1986; 7: 410-3.

25 Zhang ZD, Jin GZ, Xu SX, Yu LP, Chen Y, Jiang FY, et al. Effects of I-stepholidine on the central nervous and cardiovascular systems.
Acta Pharmacol Sin 1986; 7: 522-6.

26 Xiong ZL, Sun Z, Jin GZ, Chen Y. [Influence of I-stepholidine on blood pressure and its relation to alpha-adrenoceptors]. Acta Pharmacol Sin 1987; 8: 497-501.

27 Chu H, Jin G, Friedman E, Zhen X. Recent development in studies of tetrahydroprotoberberines: mechanism in antinociception and drug addiction. Cell Mol Neurobiol 2008; 28: 491-9.

28 Yang Z, Shao YC, Li SJ, Qi JL, Zhang MJ, Hao W, et al. Medication of I-tetrahydropalmatine significantly ameliorates opiate craving and increases the abstinence rate in heroin users: a pilot study. Acta Pharmacol Sin 2008; 29: 781-8.

29 Wang $\mathrm{H}$. Cardiovascular ATP-sensitive $\mathrm{K}^{+}$channel as a new molecular target for development of antihypertensive drugs. Acta Pharmacol Sin 1998; 19: 397-402.

30 Wang H, Zhang YL, Tang XC, Feng HS, Hu G. Targeting ischemic stroke with a novel opener of ATP-sensitive potassium channels in the brain. Mol Pharmacol 2004; 66: 1160-8.

31 Yang Y, Liu X, Long Y, Wang F, Ding JH, Liu SY, et al. Systematic administration of iptakalim, an ATP-sensitive potassium channel opener, prevents rotenone-induced motor and neurochemical alterations in rats. J Neurosci Res 2005; 80: 442-9.

32 Chen $\mathrm{H}$, Yang $\mathrm{Y}$, Yao HH, Tang XC, Ding JH, Wang $\mathrm{H}$, et al. Protective effects of iptakalim, a novel ATP-sensitive potassium channel opener, on global cerebral ischemia-evoked insult in gerbils. Acta Pharmacol Sin 2006; 27: 665-72.

33 Liu Y, He HR, Ding JH, Gu B, Wang H, Hu G. Iptkalim inhibits cocaine challenge-induced enhancement of dopamine levels in nucleus accumbens and striatum of rats by up-regulating Kir6.1 and Kir6.2 mRNA expression. Acta Pharmacol Sin 2003; 24: 527-33.

$34 \mathrm{Hu} \mathrm{J}$, Lindenberger K, Hu G, Wang H, Lukas RJ, Wu J. Iptakalim as a human nicotinic acetylcholine receptor antagonist. J Pharmacol Exp Ther 2006; 316: 914-25.

35 Hu J, DeChon J, Yan KC, Liu Q, Hu G, Wu J. Iptakalim inhibits nicotinic acetylcholine receptor-mediated currents in dopamine neurons acutely dissociated from rat substantia nigra pars compacta. Neurosci Lett 2006; 403: 57-62.

36 Yildirim MA, Goh KI, Cusick ME, Barabasi AL, Vidal M. Drug-target network. Nat Biotechnol 2007; 25: 1119-26.

37 Hopkins AL. Network pharmacology. Nat Biotechnol 2007; 25: 1110-1. 